\title{
Multinodular goiter, a cause of positional vertigo
}

\begin{abstract}
Benign Paroxysmal Positional Vertigo is a common cause of dizziness. We report a 60 year old patient with history of vertiginous episodes when she turns her head to the right sides. She was found to have a large thyroid with suprahyoid extension. The thyroid mass was pressing on the carotid while turning her neck to the right causing vertigo. Her symptoms completely resolved following right hemi-thyroidectomy. Review of literature discussed.
\end{abstract}

\author{
Volume 6 Issue I - 2017
}

\author{
Joanna Sanchez, Manoj Kumar \\ Department of Otolaryngology, CNY Medical Professionals, \\ USA
}

Correspondence: Manoj Kumar, Department of Otolaryngology, CNY Medical Professional, Cortland, NY I 3045, USA, Email mkumar@cortlandent.com

Received: October 31, 2016 | Published: January 09, 2017

\section{Introduction}

Benign Paroxysmal Positional Vertigo (BPPV) is one of the commonest causes for vertigo. The annual incidence is around $0.6 \%$ and it affects more women than men. Patients presents with history of episodic vertigo. The vertiginous attacks are triggered by a change in head position. Approximately $60 \%$ of BPPV do not have an identifiable cause. ${ }^{1}$ Other reported causes include head injury, metabolic conditions and vestibular neuritis. Several theories have been postulated to explain the pathophysiology of BPPV including canalolithiasis and cupulolithiasis. The diagnosis is often made from the clinical history and by doing a test called Dix- Hallpike test. We report a case of positional vertigo triggered by turning head to the right side. A large thyroid mass was seen pressing on the carotid when patient turns to the right. Patient's symptoms resolved following right hemi-thyroidectomy. This is the first reported case in the literature showing thyroid mass as a cause for positional vertigo.

\section{Clinical history}

A 60year old female presented to the office with history of dizziness on turning her head to right. She says she feels dizzy and spinning sensation for few seconds and it goes away when she turns her head away from the right side. Her symptoms appeared approximately 2 months ago but do not remember exactly when. Patient had symptoms while trying to reverse her car. She says when she turns her head to the right to look back she develops vertigo and had to wait few seconds before getting better. She therefore has been avoiding turning to the right. She denied nausea or vomiting. There was no history of recent hearing loss. She also did not report any other otological symptoms. Clinical examination showed an enlarged thyroid. Neuro-otological examination was essentially within normal limits except Dix- Hallpike test. She reported severe vertigo when head was down on the right side. Rotational Nystagmus was seen but the patient could not stay with her face turned to right for more than 5 seconds. She stopped feeling dizzy when the head was turned away from the right side. Epley's maneuver could not be done as the patient was unable to tolerate it. Therefore patient was advised to do Brandt Daroff exercises at home. She continued to be symptomatic despite home treatment.

Incidentally, during examination a large thyroid mass was noted. A CT scan of the neck revealed a large thyroid mass extending to suprahyoid area on the right sides. Given the size of the thyroid it was decided to do a right hemi-thyroidectomy. Patient's vertiginous symptoms completely resolved following thyroidectomy. Patient was followed up for 3 years with no recurrence of her symptoms.

\section{Discussion}

BPPV is a common cause of dizziness and is caused by abnormal stimulation of the cupula by free-floating otoliths (canalolithiasis) or otoliths that have adhered to the cupula (cupulolithiasis), mainly within the posterior semicircular canal. Patients have episodic vertigo, usually short lived and precipitated by a sudden change in head position. Dix Hallpike maneuver is the gold standard for the diagnosis of BPPV. The exact cause of BPPV is unknown in about two thirds of patients. BPPV may also develop secondary to various disorders that affect the inner ear. Head trauma is responsible for BPPV in $15-20 \%$ of cases. Vestibular neuritis, Meniere's disease and labrynthitis can lead to degeneration and detachment of otoconia. Giant cell arteritis, hyperuricemia and diabetes are also associated with BPPV.

A case series published in 2015 showed that intra-labrynthine schwannomas can produce positional vertigo without any audiological symptoms. ${ }^{2}$ Demirtas et al., ${ }^{3}$ published a case of Eagle syndrome presenting with positional vertigo. The patient in this report experienced vertigo when he turned his head to the left and the patient's complaints disappeared completely in the neutral position. A CT angiographic study confirmed that the elongated styloid process on the left side was compressing the internal carotid artery in the left cervical rotation position.

A detailed review of the published literature failed to show any case of thyroid mass presenting as positional vertigo. However, a retrospective study over a 5year period revealed five patients with intracranial pathologic conditions mimicking benign paroxysmal positional vertigo. ${ }^{4}$ These patients were first seen with episodic vertigo associated with positional change. There are also reports of patients with nasopharyngeal carcinoma presenting as syncopal attacks. ${ }^{5}$ The involvement of the glossopharyngeal or vagal nerve by para-pharyngeal extension of the tumor is postulated as the probable mechanism.

That compression of a carotid artery may induce a change in cerebral function was apparently known by the ancient Greeks. The term "carotid" is said to have been derived from the Greek word. Carotid compression may cause syncope or seizures. Carotid sinus hypersensitivity and cerebral ischemia are proposed as possible 
mechanisms. ${ }^{6}$ Hypo-perfusion to the flocculonodular lobe supplied by the anterior inferior cerebellar artery is a likely cause for the vertigo.?

Based on the case report we presented, it is recommended that patients seen with symptoms like those of benign paroxysmal positional vertigo who do not show improvement after undergoing the particle repositioning maneuver should have imaging studies of neck and brain. Measuring artery flow in neutral and neck rotated position using Doppler ultrasonography and comparing the results may be diagnostic in patients with vertigo due to vascular compression. However, the procedure is not practical because it is difficult to scan the distal part of the artery during rotation. ${ }^{3} \mathrm{CT}$ angiography may be considered an alternative option (Figure 1-3).

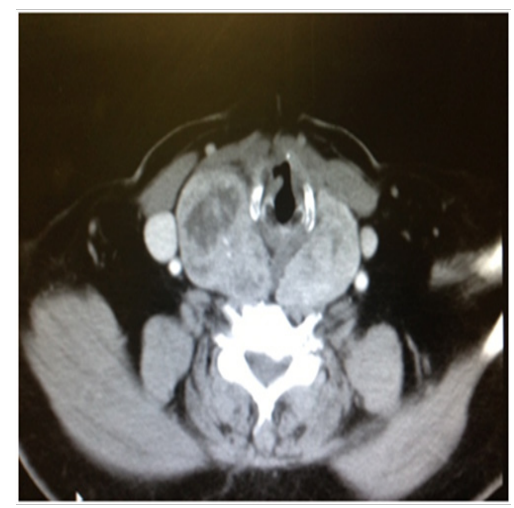

Figure I CT scan soft tissue neck showing enlarged thyroid displacing carotid artery on the right side.

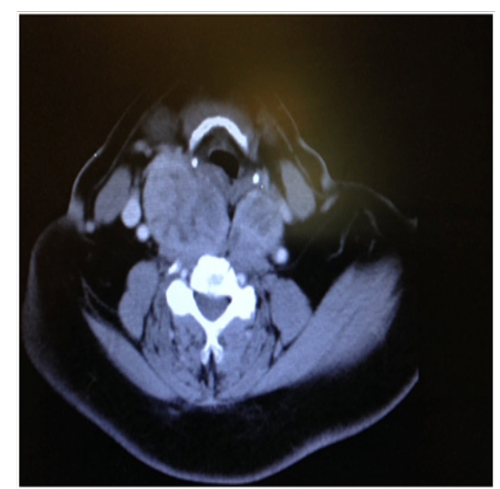

Figure $2 \mathrm{CT}$ scan soft tissue neck showing right thyroid lobe enlargement at the level of hyoid bone, again displacing carotid artery.

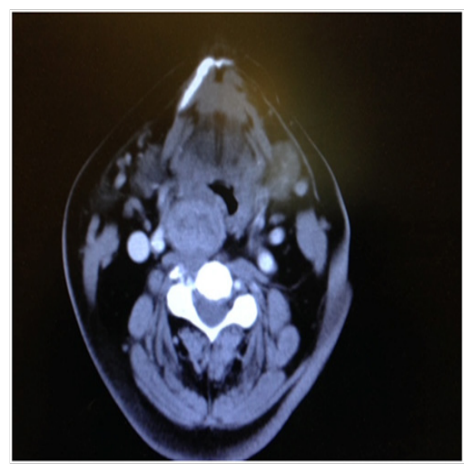

Figure $3 \mathrm{CT}$ scan soft tissue neck showing thyroid enlargement extending up to the level of mandible and base of the tongue on the right side.

\section{Acknowledgements}

None.

\section{Conflict of interest}

The author declares no conflict of interest.

\section{References}

1. Parnes LS, Agrawal SK, Atlas J. Diagnosis and management of benign paroxysmal positional vertigo (BPPV). CMAJ. 2003;169(7):681-693.

2. Slattery EL, Babu SC, Chole RA, et al. Intralabyrinthine Schwannomas Mimic Cochleovestibular disease: Symptoms From Tumor Mass Effect in the Labyrinth. Otol Neurotol. 2015;36(1):167-171.

3. Demirtaş HK, Kayan M, Koyuncuoğlu HR, et al. Eagle syndrome causing vascular compression with cervical rotation: case report. Pol J Radiol. 2016;81:277-280.

4. Dunniway HM, Welling DB. Intracranial tumors mimicking benign paroxysmal positional vertigo. Otolaryngology Head and Neck Surgery. 1998;118(4):429-436.

5. Lin RH, Teng MM, Wang SJ, et al. Syncope as the presenting symptom of nasopharyngeal carcinoma. Clin neurol Neurosurg. 1994;96(2):152155.

6. Silverstein AD, Doniger D, Bender MB. Manual compression of the carotid vessels, carotid sinus hypersensitivity and carotid artery occlusions. Ann intern med. 1960;52(1):172-181.

7. Parthasarathy R, Derksen C, Saqqur M, et al. Isolated intermittent vertigo: A presenting feature of persistent trigeminal artery. J neurosci rural pract. 2016;7(1):161-163. 